\title{
Correction to: Systemic Photoprotection
}

\author{
Jennifer H. Taylor ${ }^{1} \cdot$ Cheryl F. Rosen ${ }^{1}$ \\ Published online: 17 September 2020 \\ (C) Springer Science+Business Media, LLC, part of Springer Nature 2020
}

\section{Correction to: Curr Derm Rep (2020) 9, pp 181-188 https://doi.org/10.1007/s13671-020-00306-1}

The original version of this article did not include the results from articles showing a more favorable side effect profile with a controlled-release subcutaneous implant of afamelanotide. Updated references for these articles (as seen below) are to also be included.

Afamelanotide (Nle4-D-Phe7-a-MSH) is a synthetic analogue of $\alpha$-melanocyte stimulating hormone (MSH), a naturally occurring hormone that stimulates the production of eumelanin in the skin. Eumelanin is one of the skin's natural defenses against UV radiation, and abundant epidemiological data supports its protective role against UV-induced skin cancer [53]. An initial randomized, double-blind placebo-controlled trial using an aqueous formulation showed promising results with significantly increased melanin density in all treated participants. There was significantly reduced DNA damage and a decrease in histological evidence of UV damage after exposure to $3 \times$ the participant's MED. The side effect profile of this aqueous formulation of synthetic melanotropin included nausea, vomiting, facial flushing and fatigue [53].

The online version of the original article can be found at https://doi.org/ 10.1007/s13671-020-00306-1

\footnotetext{
Cheryl F. Rosen

crosen019@gmail.com

Jennifer H. Taylor

jennifer.taylor44@gmail.com

1 Toronto Western Hospital, University of Toronto, Toronto, ON, Canada
}

Subsequent larger cohort and controlled trials using the controlled-release subcutaneous implant in patients with erythropoietic protoporphyria showed clinical benefit with a more favorable side effect profile. There were no severe adverse effects attributed to afamelanotide and high participant retention in the treatment groups was seen with the controlledrelease formulation [54-55]. This afamelanotide implant is approved in Europe and the USA for the prevention of phototoxicity in adult patients with erythropoietic protoporphyria.

Updated References:

54. Kim E, Garnock-Jones K. Afamelanotide: a review in erythropoietic protoporphyria. Am J Clin Dermatol. 2016;17:179-185.

55. Wensink D, Wagenmakers M, Barman-Aksözen J, Friesema E, Wilson J, van Rosmalen J, Langendonk J. Association of Afamelanotide with improved outcomes in patients with erythropoietic protoporphyria in clinical practice. JAMA Dermatol. 2020;156:570-575

Publisher's Note Springer Nature remains neutral with regard to jurisdictional claims in published maps and institutional affiliations. 